\title{
miR-221 Mediates Chemoresistance of Esophageal Adenocarcinoma by Direct Targeting of DKK2 Expression
}

\author{
Yan Wang, PhD, * Yue Zhao, PhD, ${ }^{* *}$ Andreas Herbst, PhD, $\ddagger$ Thomas Kalinski, MD, § Jiwei Qin, MS, \\ Xiaoyan Wang, MS,* Zhenzhong Jiang, MD, - Frank Benedix, MD, † Sabine Franke, PhD, $\S$ \\ Thomas Wartman, PhD, $\dagger$ Peter Camaj, PhD, ${ }^{* *}$ Walter Halangk, PhD, $\dagger$ Frank T. Kolligs, MD, $\ddagger$ \\ Karl W. Jauch, MD, ${ }^{*}$ Peter J. Nelson, PhD, $\|$ and Christiane J. Bruns, MD**
}

\begin{abstract}
Background: Chemoresistance is a main obstacle to effective esophageal cancer (EC) therapy. We hypothesize that altered expression of microRNAs (miRNAs) play a role in EC cancer progression and resistance to 5-fluorouracil (5-FU) based chemotherapeutic strategies.

Methods: Four pairs of esophageal adenocarcinoma (EAC) cell lines and corresponding 5-FU resistant variants were established. The expression levels of miRNAs previously shown to be involved in the general regulation of stem cell pathways were analyzed by qRT-PCR. The effects of selected miRNAs on proliferation, apoptosis, and chemosensitivity were evaluated both in vitro and in vivo. We identified a particular miRNA and analyzed its putative target genes in 14 pairs of human EC tumor specimens with surrounding normal tissue by qRT-PCR as well as Wnt pathway associated genes by immunohistochemistry in another 45 EAC tumor samples.

Results: MiR-221 was overexpressed in 5-FU resistant EC cell lines as well as in human EAC tissue. $D K K_{2}$ was identified as a target gene for miR-221. Knockdown of miR-221 in 5-FU resistant cells resulted in reduced cell proliferation, increased apoptosis, restored chemosensitivity, and led to inactivation of the $\mathrm{Wnt} / \mathrm{\beta}$-catenin pathway mediated by alteration in $\mathrm{DKK}_{2}$ expression. Moreover, miR-221 reduction resulted in alteration of EMT-associated genes such as E-cadherin and vimentin as well as significantly slower xenograft tumor growth in nude mice. $\mathrm{RT}^{2}$ profiler analysis identified a substantial
\end{abstract}

From the *Department of Surgery, University of Munich, Munich, Germany; $\dagger$ †epartment of Surgery, Otto-von-Guericke University, Magdeburg, Germany; $\ddagger$ Department of Medicine II, University of Munich, Munich, Germany; §Department of Pathology, Otto-von-Guericke University, Magdeburg, Germany; -Department of Emergency, Guangdong Frontier Corps Hospital, Shenzhen, China; ||Clinical Biochemistry Group, Medizinische Klinik und Poliklinik IV, University of Munich, Munich, Germany; and ${ }^{* *}$ General, Visceral and Cancer Surgery, University Hospital of Cologne, Kerpener Straße 62, Cologne, Germany.

Reprints: Yue Zhao, PhD; Yan Wang, PhD; Christiane J. Bruns, MD; Department of Surgery, Otto-von-Guericke University, Leipziger Str. 44, 39120 Magdeburg, Germany. E-mail: zyberry@ gmail.com; wangyan198614@163.com; christiane.bruns@uk-koeln.de.

This study was supported by the German Research Society (DFG) grant (BR 1614/ 7-1) and the DKTK/DKFZ 2013 ("Stem cells in Oncology") to C.J.B. Yan Wang and Xiaoyan Wang were financially supported by LMU-CSC (The China Scholarship Council) scholarship.

Y.W.: experiments and data analysis, manuscript preparation; Y.Z., A.H., P.J.N., and C.J.B.: Project design, academic guidance and manuscript correction; T.K and S.F.: clinical samples collection and evaluation; J.W.Q. and X.Y.W.: experimental assistance; F.B.: clinical data update; T.W., P.C., and W.H.: scientific guidance; F.T.K., K.W.J., and C.J.B.: clinical and scientific guidance.

This study was conducted with the approval of the Otto-von-Guericke University Hospital Research ethics committee and informed consent was obtained according to the committee's regulations and the Declaration of Helsinki.

The authors report no conflicts of interest.

Supplemental digital content is available for this article. Direct URL citations appear in the printed text and are provided in the HTML and PDF versions of this article on the journal's Web site (www.annalsofsurgery.com).

Copyright (c) 2016 Wolters Kluwer Health, Inc. All rights reserved.

ISSN: 0003-4932/16/26405-0804

DOI: $10.1097 /$ SLA.0000000000001928 dysregulation of $4 \mathrm{Wnt} / \beta$-catenin signaling and chemoresistance target genes as a result of miR-221 modulation: $C D H 1, C D 44, M Y C$, and $A B C G 2$.

Conclusion: MiR-221 controls 5-FU resistance of EC partly via modulation of Wnt/ $\beta$-catenin-EMT pathways by direct targeting of $\mathrm{DKK}_{2}$ expression. MiR-221 may serve as a prognostic marker and therapeutic target for patients with 5-FU resistant EAC.

Keywords: 5-FU resistance, epithelial-mesenchymal transition, esophageal cancer, miR-221, Wnt/ $\beta$-catenin signaling

(Ann Surg 2016;264:804-814)

E sophageal cancer (EC) is the eighth most common cancer and the E sixth most common cause of cancer death. ${ }^{1}$ The current clinical treatment options for EC are surgery, chemotherapy, and radiotherapy. The prognosis of patients receiving surgery alone is still poor. ${ }^{2}$ Multimodal treatment improves the survival. Survival rates following esophagectomy with added neoadjuvant therapy are increasing to $30 \%$ to $45 \%$ for 5 -year survival. ${ }^{3}$ However, the response rate to chemotherapy, including 5-FU, is still lower than $50 \%{ }^{4}$ Chemoresistance is thus seen as a major obstacle in the effective treatment of EC. The 2 main subtypes of the disease are esophageal squamous-cell carcinoma (often abbreviated to ESCC), which is more common in the developing world, and esophageal adenocarcinoma (EAC) is more common in the European and north American countries,${ }^{5}$ highly associated with smoking tobacco, obesity, and acid reflux. $^{6}$

MicroRNAs (miRNAs) are small 19 to 22nt noncoding single-strand RNAs that mediate gene expression by interacting with the $3^{\prime}$ UTR regions of their target gene mRNA and blocking its translation. ${ }^{7}$ MiRNAs have been shown to regulate diverse cellular processes, including cell proliferation, stemness and differentiation, apoptosis, as well as therapy resistance. ${ }^{8}$ Accumulating evidence suggests that miRNAs are dysregulated in a variety of cancers including EC. Cancer chemoresistance is a complicated process manifested through multiple mechanisms, including DNA damage repair, expression of ATP-binding cassette drug transporters, and activation of PI3K/AKT and Wnt pathways. ${ }^{9-13}$ In addition, micro-environmental stimuli such as tissue hypoxia or signals that influence epithelial-mesenchymal transition can also impact chemoresistance. ${ }^{9-13}$ Recent reports have described miR141, miR-200c, miR-148a, miR-296, and miR-27a as functionally contributing to chemoresistance of EC. ${ }^{14-19}$ Overexpression of miR-200c was shown to induce resistance in ECs through activation of the Akt signaling pathway. ${ }^{15}$ However, the molecular mechanism underlying this observation remains unclear. In the present study, we show that miR-221 expression is significantly increased in 5-FU resistant EC cell lines and the underlying molecular mechanism was further elucidated in a series of in vitro and in vivo experiments. 


\section{METHODS}

\section{Cell Culture and Establishment of Chemotherapy- Resistant EC Cell Lines}

The OE19 and OE33 human EC cell lines were obtained from the Sigma Cell Line Bank (Sigma, 96071721 and 96070808). Two additional human EC cell lines, PT1590 and LN1590, were provided by the University Medical Center of Hamburg-Eppendorf. All cell lines were maintained in culture as described previously. ${ }^{19} 5$-FU resistant EC cell lines $\left(\mathrm{OE} 19-5 \mathrm{Fu}_{\text {res }}, \mathrm{OE} 33-5 \mathrm{Fu}_{\text {res }}, \mathrm{PT}-5 \mathrm{Fu}_{\mathrm{res}}\right.$, and $\mathrm{LN}-5 \mathrm{Fu}_{\mathrm{res}}$ ) were developed through a stepwise incremental treatment with 5-FU as follows: the initial concentration of 5-FU used was set at $5 \mu \mathrm{g} / \mathrm{mL}$ on the basis of IC50 values ranging between 3 and $6.5 \mu \mathrm{g} /$ $\mathrm{mL}$ for the original sensitive cell lines. After 24 hours, the cells were passaged with 5-FU free medium. Upon reaching confluency, the cells were treated with increasing levels of 5-FU (1.5- to 2-fold). After 5 subsequent steps with increasing 5-FU concentration, the resistant cell lines were eventually established.

\section{Tissue Specimens}

Tissue samples were obtained from patients with EAC between 2005 and 2015 at the Department of Surgery, University of Magdeburg, Germany (Ethic Committee approval 33/01, University of Magdeburg, Magdeburg, Germany). The description of these specimens is detailed in supplementary table S1, http://links.lww.com/SLA/B69.

\section{MiRNA and Gene Expression Analysis}

Total RNA from fresh-frozen esophageal tumor tissues or cell lines was isolated using QIAzol lysis reagent (Qiagen, USA). Expression of let-7b, let-7g, miR-21, miR-34a, miR-92, miR200c, and miR-221 was determined using miScript SYBR Green PCR kits (Qiagen, USA). Expression of RNU6B (Qiagen, USA) was used as endogenous control. miRNAs sequences are provided in the supplementary table S2, http://links.lww.com/SLA/B69. For determining steady-state mRNA expression, total RNA was reverse transcribed to cDNA and qRT-PCR was performed as described previously. ${ }^{20}$ Primer sequences are provided in supplementary table S3, http://links.lww.com/SLA/B69.

\section{Transfection of miR-221 Mimics, miR-221 Inhibitor, and CTNNB1 siRNA}

To transiently modulate miR-221 expression, hsa-miR-221 mimics (Qiagen, Cat. no: MSY0000278, USA), hsa-miR-221 inhibitor (Qiagen, Cat. no: MIN0000278, USA), or negative control siRNA (Qiagen, Cat. no: 1022076, USA) were transfected into 5-FU sensitive or resistant EC cells. ${ }^{20}$ To suppress CTNNB1, expression cells were transfected with either CTNNB1 siRNA (GE Healthcare, Cat.no: M-003482-00-0005) or negative control siRNA using HiperFect transfection reagent (Qiagen, Cat.no:301705, USA). Sequences for the hsa-miR-221 mimics, hsa-miR-221 inhibitor, and CTNNB1 siRNA are provided in Table S4.

\section{TOP Flash Luciferase Report Assay}

To assess TCF- $\beta$-catenin mediated transcriptional activity, hsa-miR-221 mimics/inhibitor or siR-CTNNB1 and/or the $8 \times$ TOP-flash reporter gene construct were cotransfected into cells, and TOP-flash luciferase reporter gene assays were performed using a Luciferase Reporter Assay System (Promega, Madison, WI). ${ }^{21}$

\section{Protein Analysis}

Western blotting was performed as described previously. ${ }^{22}$ Antibody information is provided in supplemental materials, http://
links.lww.com/SLA/B69. The protein bands were visualized using a Leica DFC450D fluorescence microscope (Leica, Wetzlar, Germany).

\section{Cell Proliferation, Apoptosis, and Chemosensitivity}

Regarding cell proliferation, EC cells were analyzed at 0 , 24, 48, and 72 hours after transfection with hsa-miR-221 mimics (miR-221), hsa-miR-221 inhibitor (anti221), or negative control siRNA (siRCtrl) using the Cell Counting kit-8 (CCK-8) (Dojindo Laboratories, Kumamoto, Japan). Annexin V-fluorescein isothiocyanate (FITC) and propidium iodide (PI) staining (Miltenyi Biotec, Germany) were applied to determine the percentage of transfected cells undergoing apoptotic or necrotic cell death after 24 hours. To assess cytotoxicity of 5-FU, all transfected cells were analyzed at 48 hours following 2.5 or $20 \mu \mathrm{g} / \mathrm{mL}$ 5-FU treatment.

\section{Esophageal Adenocarcinoma Xenograft Mouse Model}

To determine tumor growth in vivo, EC cells transfected with miR-221, anti221, or siRCtrl were implanted into the flanks of 6 to 8week-old male Balb/c nu-nu mice (Charles River Deutschland, Sulzfeld, Germany). Tumor size was measured in specified time intervals. Upon sacrifice of the animals, tumor volume was calculated and analyzed. All protocols were approved by the regional commission for animal experiments in the state of Sachsen-Anhalt of Germany (no.42502-2-1266 uniMD).

\section{Histology and Immunostaining}

For immunohistochemical staining samples from the in vivo animal experiments, patient-derived esophageal tumor or adjacent normal tissues were fixed, embedded and sectioned at $3 \mu \mathrm{m}$ thickness. These sections were then stained with anti-Ki67 $(1: 100$, Abcam, UK), anti- $\beta$-catenin $(1: 500$, BD Bioscience, Cat.no: 610154, USA), and anti-DKK 2 (1:200, Abcam, UK). ${ }^{20}$ Frozen tumor tissues embedded in O.C.T at $-20^{\circ} \mathrm{C}$ and sectioned to $3 \mu \mathrm{m}$ thickness were used for immunofluorescent staining with anti-CD31 (1:50, Abcam, UK).

\section{$\mathrm{RT}^{2}$ Profiler PCR Array System}

The expression of selected Wnt/ $\beta$-catenin-EMT related genes was examined using the Custom Human RT $^{2}$ Profiler TM PCR array (RT ${ }^{2}$ Profiler TM PCR Array: CAPH12950), which includes targets of the Wnt/ $/$-catenin signaling pathway as well as genes associated with the general process of EMT. Total RNA was isolated from $5 \times 10^{5}$ OE33 and OE33-5Fu res $_{\text {en }}$ cells with or without hsa-miR-221 mimics, inhibitor, or siR-CTNNB1 using the miRNeasy kit. PCR was performed with the $\mathrm{RT}^{2}$ profiler PCR array system and analyzed using an ABI 7000 PCR machine. The expression levels of different mRNAs were normalized using a series of housekeeping genes: $A C T B, B 2 M, G A P D H, H P R T 1$, and RPLPO. The fold change of gene expression from the different treatment as compared with control groups was calculated as $2^{(-\Delta \Delta C T)}$.

\section{Statistical Analysis}

All data are expressed as mean \pm SD. The correlation of $\beta$ catenin or $\mathrm{DKK}_{2}$ and each clinical pathologic variable was comparatively analyzed by $\chi^{2}$ test and the Fisher exact test. A $P$ value of less than 0.05 indicated the presence of statistically significant difference between groups. All statistical analyses were carried out with Graphpad Prism 6. For $\mathrm{RT}^{2}$ profiler data analysis, we applied a web-based service of Qiagen data analysis center. Genes were called "differentially expressed" if the corrected $P$ value was less than 0.05 . 

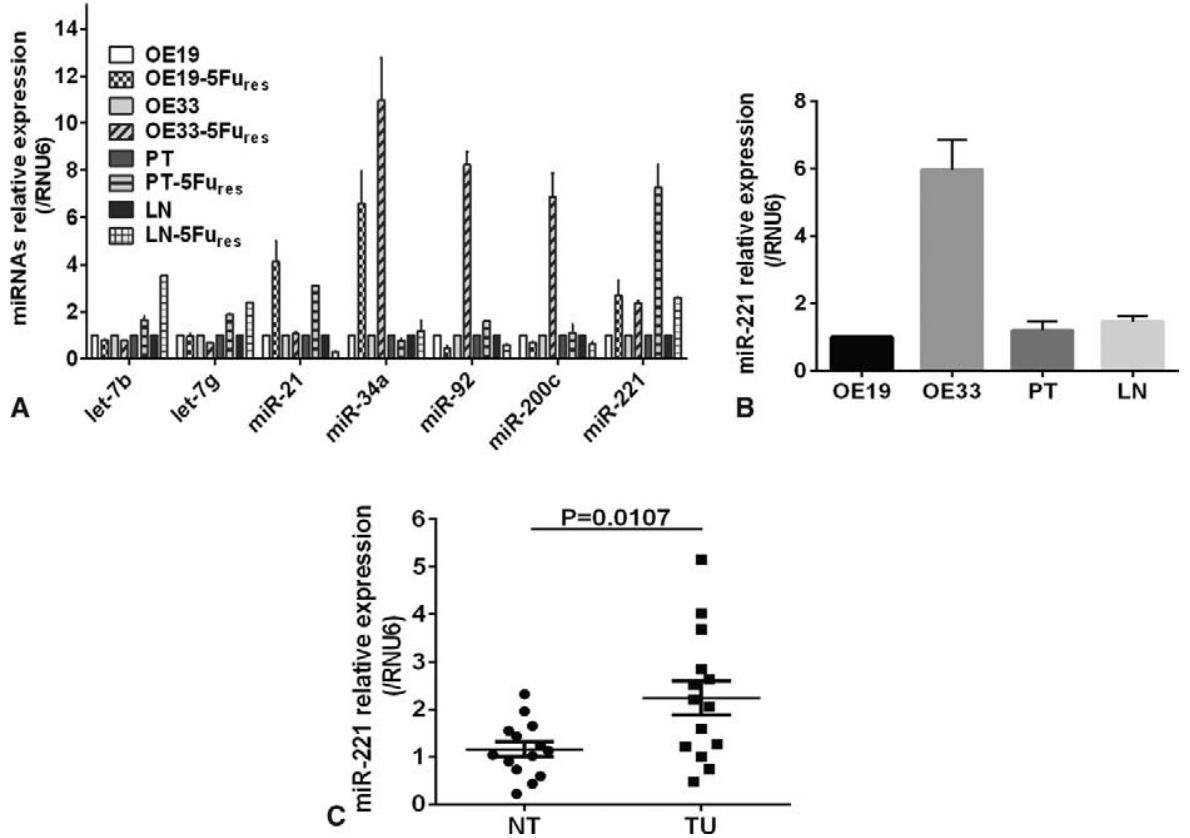

\section{RESULTS}

\section{miR-221 is Upregulated in 5-FU Resistant EC Cells} Lines and Tumor Tissues

The biology underlying stemness has been linked to the biology of chemoresistance. ${ }^{23-25}$ A series of miRNAs have been previously implicated in the regulation of "stem cell" function. ${ }^{26,27}$

To study the role of miRNAs in the context of chemotherapy resistance in $\mathrm{EC}$, we established a series of 5-FU resistant $\mathrm{EC}$ cell lines $\left(\mathrm{OE} 19-5 \mathrm{Fu}_{\mathrm{res}}, \mathrm{OE} 33-5 \mathrm{Fu}_{\mathrm{res}}, \mathrm{LN}-5 \mathrm{Fu}_{\mathrm{res}}\right.$, and PT-5Fu $\mathrm{res}_{\text {res }}$ ) (Fig. S1, http://links.lww.com/SLA/B69) and then analyzed them for their differential expression of miRNAs by RT-qPCR. In a panel of 5-FU resistant EAC cells (OE19, OE33, PT1590, and LN1590), miR-221 was overexpressed in all resistant variants (Fig. 1A). The highest expression of miR-221 was found in the OE33 cell line (Fig. 1B). However, we did not show all miRNA transfection data in the other 3 cell lines; OE19-5FU res did not display an ideal growth under transfection. Therefore, $\mathrm{OE} 33$ and $\mathrm{OE} 33-5 \mathrm{Fu}_{\text {res }}$ cells were selected for the subsequent functional studies. MiR-221 was further validated in EC patient's samples. MiR-221 was significantly higher expressed in tumor than in adjacent normal tissue from $14 \mathrm{EC}$ patients (Fig. 1C, $P=0.0107)$.

\section{Inhibition of miR-221 in EC Cells Decreases Cell Proliferation, Induces Apoptotic Cell Death, and Restores 5-FU Sensitivity}

To investigate the role of miR-221 on proliferation, cell death, and chemosensitivity for $\mathrm{EC}, \mathrm{OE} 33-5 \mathrm{Fu}_{\text {res }}$ cells were transiently transfected with has-miR-221 mimics (miR-221), miR-221 inhibitor (anti-221), or negative control siRNA (siRCtrl) (Fig. 2A). Transfection of miR-221 inhibitor (anti-221) significantly reduced cell viability (Fig. 2B) and increased the proportion of apoptotic cells (Figs. 2C, D) as compared with negative control siRNA in $\mathrm{OE} 33-5 \mathrm{Fu}_{\text {res }}$ cells $(45.63 \pm 2.76 \%$ vs $5.44 \pm 1.26 \%$ at 24 hours, $P<0.001)$.
FIGURE 1. miRNA expression in esophageal cancer cell lines and patient tissues. A, Relative expression of selected miRNAs assessed in 4 pairs of 5-FU sensitive and resistant esophageal cancer cells by qPCR. B, miR-221 expression in 4 esophageal adenocarcinoma cell lines. $C$, miR221 expression in 14 sets of corresponding tumor and nontumor specimens derived from esophageal cancer patients. NT indicates nontumor; TU, tumor.
Transient knockdown of miR-221 expression was found to restore sensitivity of $\mathrm{OE} 33-5 \mathrm{Fu}_{\text {res }}$ cells to 5 -FU leading to an increasing percentage of dead $\mathrm{OE} 33-5 \mathrm{Fu}_{\mathrm{res}}$ cells after re-introduction of 2.5 and $20 \mu \mathrm{g} / \mathrm{mL} 5$-FU treatment (4.7-fold and 1.5-fold, Fig. 2E).

In addition, the protein expression of thymidylate synthase (TS), a known 5-FU target and resistance marker, was reduced by transient miR-221 knockdown in $\mathrm{OE} 33-5 \mathrm{Fu}_{\mathrm{res}}$ as evidenced by western blotting (Fig. 2F).

\section{Knockdown of miR-221 Inhibits Tumor Growth In Vivo}

BALB/c nu-nu male mice were obtained from Charles River Deutschland (Sulzfeld, Germany) at 6 to 8 weeks of age and housed in the animal facility of the University Medical Center Magdeburg. Thirty mice were randomized into 6 groups ( 5 mice per group). EC tumor xenografts were established by subcutaneous injection of $1 \times 10^{6}$ of OE33 or OE33-5Fu $\mathrm{Fu}_{\text {res }}$ following transfection with has-miR-221 mimics, miR-221 inhibitor, or negative control siRNA in the same position on the flanks of nude mice. Sixty-six days after injection, all mice were sacrificed and the harvested tumors were analyzed. Knockdown of miR-221 in $\mathrm{OE} 33-5 \mathrm{Fu}_{\text {res }}$ cells led to a significantly slower tumor growth in vivo than controls (anti-221 vs Ctrl or siRCtrl, $P<0.001$, Fig. 3C). Immunohistochemical analysis revealed that Ki67 was significantly weaker expressed in the anti-221 transfected OE33$5 \mathrm{Fu}_{\text {res }}$ cells than the controls (anti-221 vs Ctrl or siRCtrl, $P<0.0001$ ) (Fig. 3E). CD31 was performed to analyze effects on tumor angiogenesis. $\mathrm{OE} 33-5 \mathrm{Fu}_{\text {res }}$ control tumors revealed a high number of $\mathrm{CD} 31$ positive cells, which was substantially reduced in anti-221 transfected OE33-5Fu $\mathrm{res}_{\text {res }}$ tumors $(P<0.05)($ Fig. $3 \mathrm{~F})$.

\section{$\mathrm{DKK}_{2}$ is a Direct Target of miR-221 and miR-221 Induced Chemoresistance is Mediated Through the Wnt/ $\beta$-catenin Signaling}

To search for target genes of miR-221, we used 4 miRNA target prediction tools: TargetScan, PITA, miRTarBase, and miRanda 

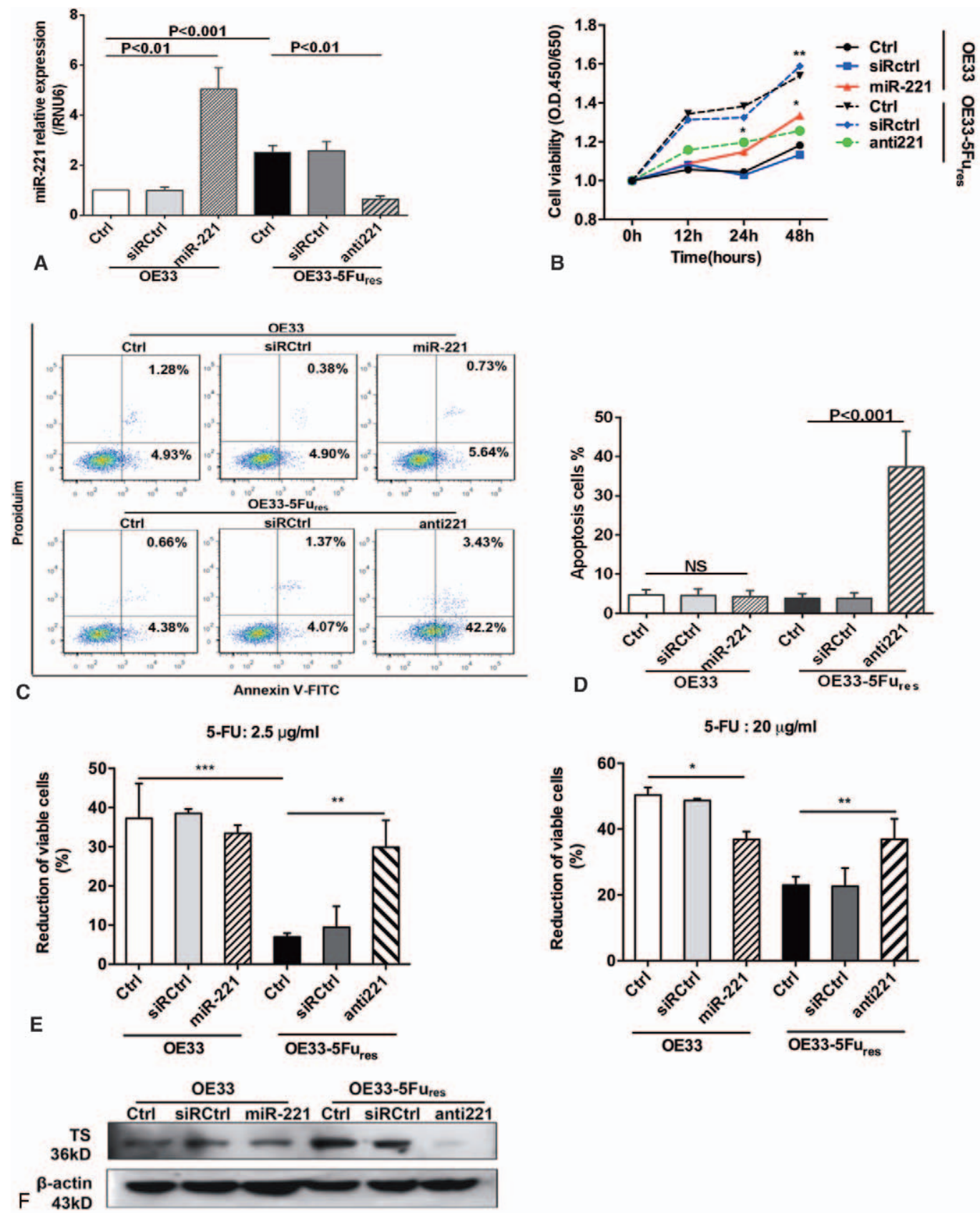

FIGURE 2. Functional analysis of miR-221 in 5-FU resistant EC cells. A, Efficacy of has-miR-221 mimics, has-miR-221 inhibitor in EC cells. Transfection with miR-221 mimics increases miR-221 expression in 5-FU sensitive EC cell (OE33), while transfection with miR221 inhibitor decreases miR-221 expression in 5-FU resistant EC cell (OE33-5Fures). B, Cells were transfected and their viability determined by CCK-8 assay at $0,24,48$, and 72 hours. The viability of control cells, cells transfected with miR-221 mimics (miR221), inhibitor (anti221), and negative control siRNA (siRCtrl) was detected. Inhibition of miR-221 expression led to reduction of cell proliferation. ${ }^{*} P<0.05, \dagger P<0.01$, 2-way ANOVA. C, Analysis of cell apoptosis of 5-FU sensitive and resistant OE33 cells at 24 hours after transfection. Overexpression of miR-221 had no impact on the cell apoptosis of 5-FU sensitive EC cells, while inhibition of miR221 significantly promoted the cell apoptosis of 5-FU resistant EC cells. D, Annexin V-positive cells were quantified by flow cytometry. Data are presented as mean SD of 3 independent experiments. E, Cell response to 5 - $\mathrm{FU}(2.5 \mathrm{and} 20 \mu \mathrm{g} / \mathrm{mL})$ after transfection. With $20 \mu \mathrm{g} / \mathrm{mL}$ of $5-\mathrm{FU}$, treatment-naive OE33 cells showed a $50.3 \% \pm 4.1 \%$ reduction of viable cells of as compared with $36.9 \% \pm 4.1 \%$ in miR-221 mimic transfected cells $(P<0.05)$, while in OE33-5FU res cells, reduction of viable cells was significantly increased from $23.0 \% \pm 4.6 \%$ to $37.0 \% \pm 8.7 \%(P<0.01)$. F, Protein expression of 5-FU resistant marker TS in OE33 and OE33- $5 \mathrm{Fu}_{\text {res }}$ cells. Transfection with miR-221 mimics slightly increased TS protein expression, while transfection with miR-221 inhibitor significantly decreased TS protein level. 

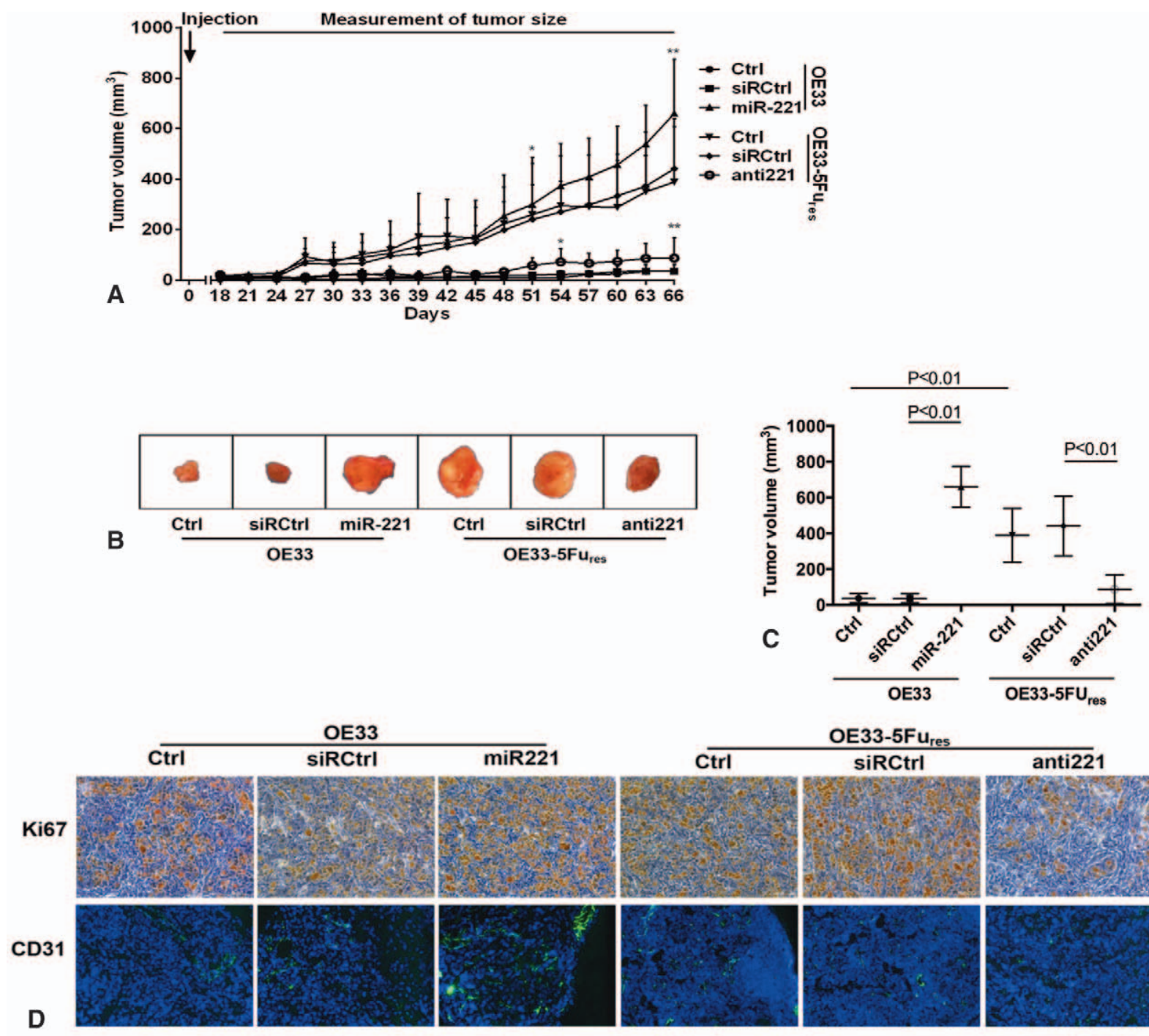

OE33-5Fu $\mathrm{u}_{\text {res }}$

siRCtrl anti221
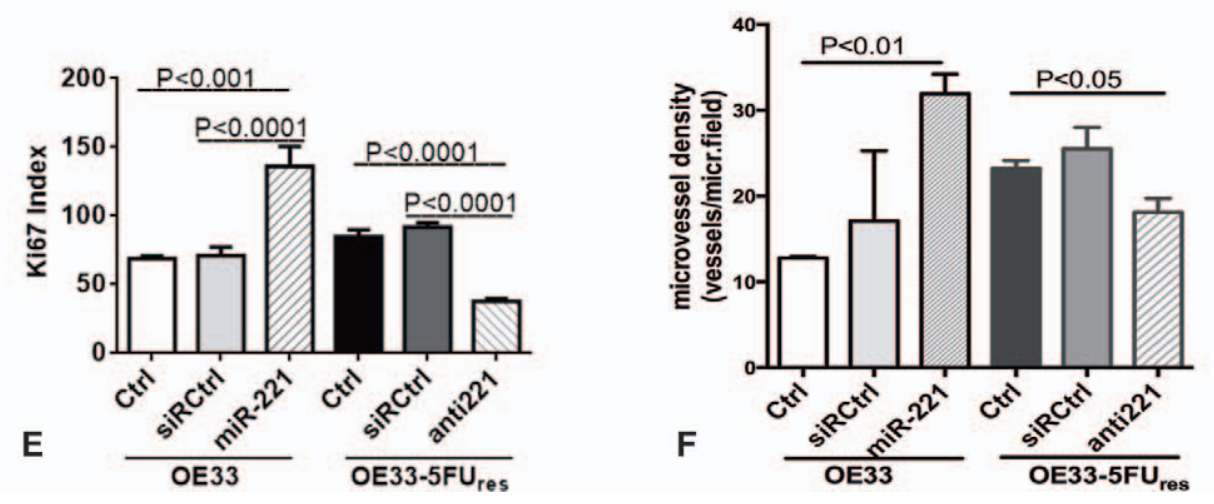

FIGURE 3. Analysis of in vivo EC tumorigenesis in a xenograft nude mouse model. A-C, Increased expression of miR-221 led to enhanced OE33 tumor growth in a xenograft nude mouse model, knockdown of miR-221 expression inhibited OE33-5FU res $_{\text {tumor }}$ growth. ${ }^{*} P<0.05, \dagger P<0.01,2$-way ANOVA. D-F, Immunohistochemical analysis of cell proliferation and immunofluorescence staining of angiogenesis. In tumors with miR-221 overexpression, Ki67 expression was significantly higher than in control groups $(P<0.0001)$. In tumors with low levels of miR-221 of OE33-5FU res after antagomir therapy, the expression of Ki67 was significantly reduced $(P<0.0001)$. CD31 expression was dramatically increased in miR-221 transfected OE33 $(P<0.01)$ and decreased in OE33-5FU res tumor with anti-miR-221 therapy $(P<0.05)$. 

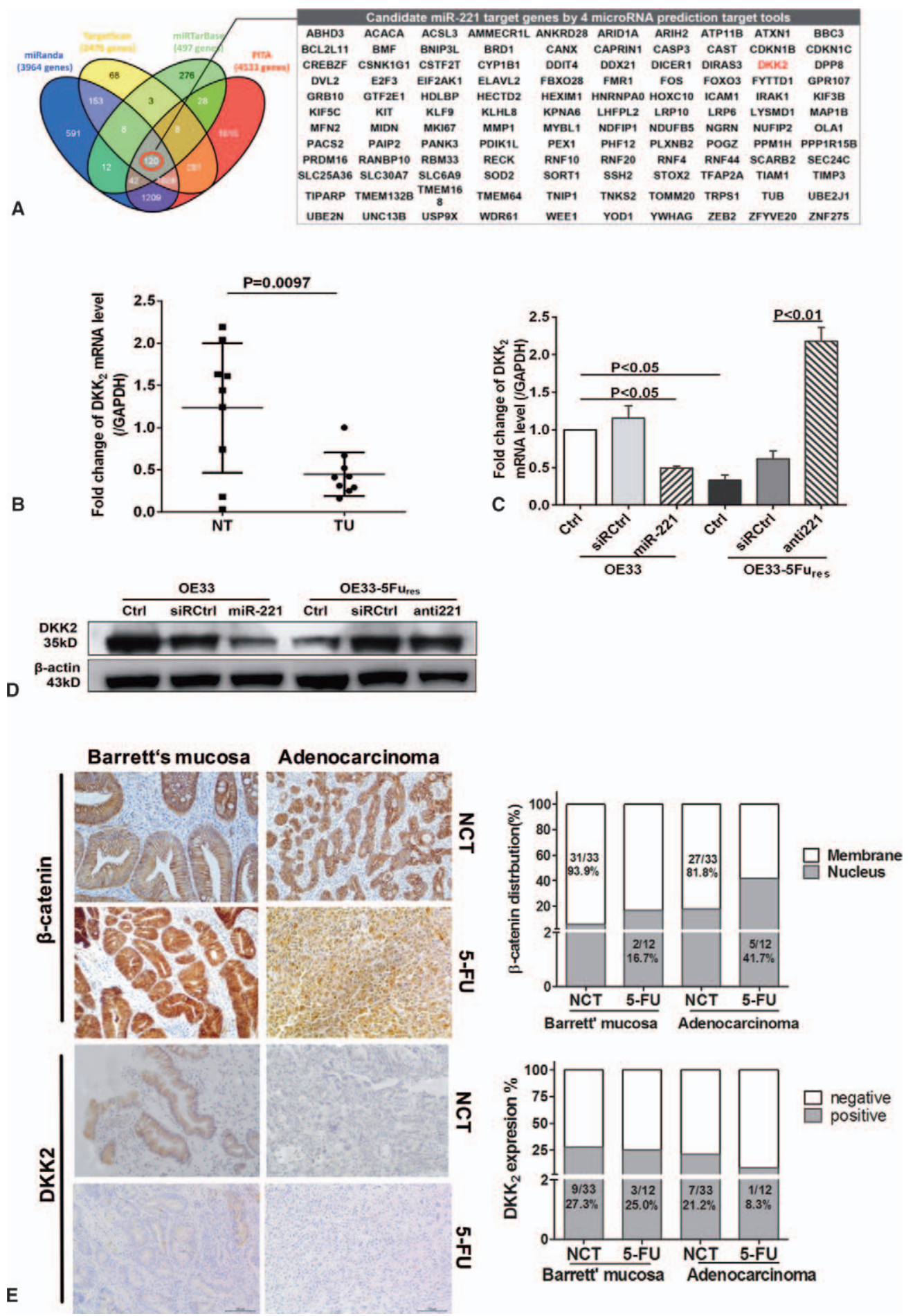

FIGURE 4. Identification of miR-221 target genes and its regulation of the Wnt/ $\beta$-catenin signaling pathway (A) Prediction of miR221 target genes. One hundred twenty target genes that have miR-221 seed sites are predicted via 4 different miRNA target prediction tools (TargetScan, PITA, miRTarBase, and miRanda). B-D, DKK 2 expression in esophageal tumor tissue and esophageal cancer cells. DKK 2 was substantially lower expressed in esophageal tumors than in adjacent nontumor tissues. Furthermore, DKK 2 was downregulated in 5-FU resistant EC cells compared with the respective sensitive cells. Overexpression of miR-221 suppressed mRNA (detected by qRT-PCR) and protein (detected by western blot) expression of DKK 2 while downregulation of miR-221 increased both $\mathrm{DKK}_{2}$ mRNA and protein expression. E, Distribution of $\beta$-catenin in esophageal tumor tissues. Nuclear localization of $\beta$-catenin in 5-FU treated esophageal tumors. 5-FU indicates 5-fluoruracil; NCT, no chemotherapy. 
(Fig. 4A). Among a large number of potential targets based on bioinformatic investigations, we identified $\mathrm{DKK}_{2}$ for further functional analysis. A potential targeting of the $3^{\prime}$ UTR of the human $D K K_{2}$ gene by miR-221 was predicted by the TargetScan and RNAhybrid software and further supported by the high level of evolutionary conservation of the seed sequence between species (data were not shown).

qPCR analysis showed that $\mathrm{DKK}_{2}$ mRNA levels were reduced in tumor samples as compared with nontumor tissue samples and in 5-FU resistant as compared with 5-FU sensitive EC cells. Thus, expression of $\mathrm{DKK}_{2}$ is inversely correlated to expression of miR-221 (Figs. 1C, 4B). However, due to the sample size, the statistics of correlation analysis between miR-221 and $\mathrm{DKK}_{2}$ mRNA expression is not significant $(P=0.216, R=0.339$; nonparametric spearman correlation). In addition, miR-221 knockdown resulted in a significant increase in $\mathrm{DKK}_{2}$ mRNA and protein expression (Figs. 4C, D).

To verify that $\mathrm{DKK}_{2}$ is a direct target of miR-221, luciferase reporter vectors were then applied. Overexpression of miR-221 dramatically decreased the luciferase reporter activity when the wild-type $\mathrm{DKK}_{2} 3^{\prime} \mathrm{UTR}$ was used, but did not influence reporter expression when the $\mathrm{DKK}_{2}$ mutant control construct containing mutant seed sequences was used (data were not shown).

To investigate a potential association between miR-221 expression, chemoresistance, and activation of the $\mathrm{Wnt} / \beta$-catenin signaling pathway, $\beta$-catenin distribution and $\mathrm{DKK}_{2}$ expression were detected in tumor samples. We found no significant difference in $\mathrm{DKK}_{2}$ expression in Barrett's mucosa $(9 / 33,27.3 \%)$ or tumor tissues $(7 / 33,21.2 \%)$ before chemotherapy. However, samples from esophageal tumor patients with prolonged exposure to chemotherapy showed a substantial decrease in $\mathrm{DKK}_{2}$ expression $(1 / 12,8.3 \%)$ as compared with the corresponding expression level seen in adjacent Barrett's mucosa $(3 / 12,25.0 \%)$.

Tumor samples following chemotherapy also demonstrated evidence of enhanced canonical Wnt signaling. Although inactivated $\beta$-catenin was mainly localized to the cell membrane and cytoplasm in Barrett's mucosa $(31 / 33,93.9 \%)$ and EAC specimens without chemotherapy treatment $(27 / 33,81.8 \%)$, an accumulation of nuclear $\beta$-catenin was found in EAC after long-term exposure to 5-FU based chemotherapy $(5 / 12,41.7 \%)$ (Fig. 4E). Among all available clinical and pathological parameters, only $\mathrm{DKK}_{2}$ expression was found to significantly correlate with the stage of tumor differentiation $(P=0.024$, Table 1).

To validate the functional role of miR-221 regulating $\beta$-catenin activation, $\mathrm{OE} 33-5 \mathrm{Fu}_{\mathrm{res}}$ cells were transfected with has-miR-221 mimics, inhibitor, siR-CTNNB1/siR- $\beta$-catenin, or negative control siRNA (Fig. 2A, S2B and C, http://links. lww.com/SLA/B69). TOP Flash luciferase reporter analysis showed decreased luciferase activity following transfection with miR-221 inhibitors equivalent to siR-CTNNB1/siR- $\beta$-catenin $\left(^{* *} P<0.01\right.$, Fig. S3B, http://links.lww.com/SLA/B69). These results suggest that miR-221 influences chemoresistance at least in part through activation of the $\mathrm{Wnt} / \beta$-catenin pathway via direct control of $\mathrm{DKK}_{2}$ expression.

\section{miR-221 Mediates EMT Through Activating Wnt/ $\beta$-catenin Signaling}

EMT is associated with chemoresistance in various cancers. We observed a morphological change toward a mesenchymal cell phenotype in 5-FU resistant EC cells (Fig. 5A). Western blot analysis showed that the epithelial cell marker E-cadherin was expressed at a lower level, whereas the mesenchymal cell marker Vimentin was expressed at a higher level in $\mathrm{OE} 33-5 \mathrm{Fu}_{\text {res }}$ cells than the respective sensitive EC cells. These results suggest that the process of EMT is associated with the development of 5-FU resistance in EC cells. Interestingly, a knockdown of miR-221 expression in $\mathrm{OE} 33-5 \mathrm{Fu}_{\text {res }}$ resulted in a significant increase in E-cadherin and a decrease in Vimentin protein expression (Fig. 5B).

To validate downstream effects of miR-221 expression on regulation of $\mathrm{Wnt} / \beta$-catenin signaling and EMT, we identified $30 \beta$ catenin target genes related to EMT by PCR array (Fig. 5C, Table S6, http://links.lww.com/SLA/B69). Four genes $M Y C, C D 44, A B C G 2$, and $C D H 1$ were significantly dysregulated between OE33 and $\mathrm{OE} 33-5 \mathrm{Fu}_{\mathrm{res}}$, following induction or inhibition of miR-221 (Fig. 5D).

\section{DISCUSSION}

MiR-221 is located on chromosome $\mathrm{X}$ and is overexpressed in osteosarcoma, ${ }^{28}$ colorectal, ${ }^{29}$ ovarian, ${ }^{30}$ breast, ${ }^{31}$ and pancreatic cancer. ${ }^{20}$ One current study showed that miR-21, miR-143, miR203, miR-205, and miR-221 were overexpressed in squamous-cell carcinoma cancer tissues compared with normal esophageal tissues. $^{32}$ In colorectal cancer, miR-221 promotes cell proliferation. ${ }^{29}$ In addition, miR-221 plays a role in mediating radio-chemoresistance of various cancers by targeting various signal transduction pathways. $^{28,31,33,34}$ Targeting of the PI3K/Akt signaling axis by miR-221 can induce cell proliferation and BCNU resistance in human glioblastoma. ${ }^{35}$ Downregulation of miR-221 also alters radiation sensitivity in these cells by targeting the PTEN pathway. ${ }^{36}$ Overexpression of miR-221 is associated with tamoxifen resistance in breast cancer through its negative regulation of estrogen receptor alpha. ${ }^{31}$ Furthermore, miR-221 has been shown to confer breast

TABLE 1. $\beta$-catenin Localization and DKK2 Expression in Association With Clinical and Pathological Parameters in Human Esophageal Tumor Samples

\begin{tabular}{|c|c|c|c|c|c|c|}
\hline Feature & $\begin{array}{c}\beta \text {-catenin } \\
\text { (Cell Membrane) } \\
\mathrm{n}=\mathbf{3 4}\end{array}$ & $\begin{array}{c}\beta \text {-catenin } \\
\text { (Cytoplasm and Nucleus) } \\
n=11\end{array}$ & $\boldsymbol{P}$ & $\begin{array}{c}\text { DKK }_{\mathbf{2}} \text { Negative } \\
\mathbf{n}=\mathbf{3 7}\end{array}$ & $\begin{array}{c}\mathrm{DKK}_{\mathbf{2}} \text { Positive } \\
\mathbf{n}=\mathbf{8}\end{array}$ & $\boldsymbol{P}$ \\
\hline Age $($ mean $\pm S D), y$ & $67.6 \pm 11.4$ & $70 \pm 10.2$ & 0.536 & $67.0 \pm 10.4$ & $72.8 \pm 13.1$ & 0.179 \\
\hline Differentiation & & & 0.619 & & & 0.024 \\
\hline Well differentiated & $7(20.6 \%)$ & $1(9.1 \%)$ & & $8(21.6 \%)$ & $0(0 \%)$ & \\
\hline Moderately differentiated & $14(41.2 \%)$ & $6(54.5 \%)$ & & $13(35.1 \%)$ & $7(87.5 \%)$ & \\
\hline Poorly differentiated & $13(38.2 \%)$ & $4(36.4 \%)$ & & $16(43.2 \%)$ & $1(12.5 \%)$ & \\
\hline Stage $(\mathrm{I}+\mathrm{II} / \mathrm{III}+\mathrm{IV})$ & $22 / 12$ & $5 / 6$ & 0.257 & $23 / 14$ & $4 / 4$ & 0.524 \\
\hline pT (T1/T2/T3/T4) & $16 / 4 / 14 / 0$ & $7 / 2 / 2 / 0$ & 0.379 & $19 / 6 / 12 / 0$ & $5 / 0 / 3 / 0$ & 0.472 \\
\hline $\mathrm{pN}(\mathrm{N} 0 / \mathrm{N} 1 / \mathrm{N} 2 / \mathrm{N} 3)$ & $20 / 10 / 0 / 4$ & $8 / 2 / 1 / 0$ & 0.169 & $22 / 10 / 1 / 4$ & $6 / 2 / 0 / 0$ & 0.718 \\
\hline Chemotherapy (5-Fu based) & & & 0.136 & & & 0.419 \\
\hline Yes & $7(20.6 \%)$ & $5(45.5 \%)$ & & $11(29.7 \%)$ & $1(12.5 \%)$ & \\
\hline No & $27(79.4 \%)$ & $6(54.5 \%)$ & & $26(70.3 \%)$ & $7(87.5 \%)$ & \\
\hline
\end{tabular}



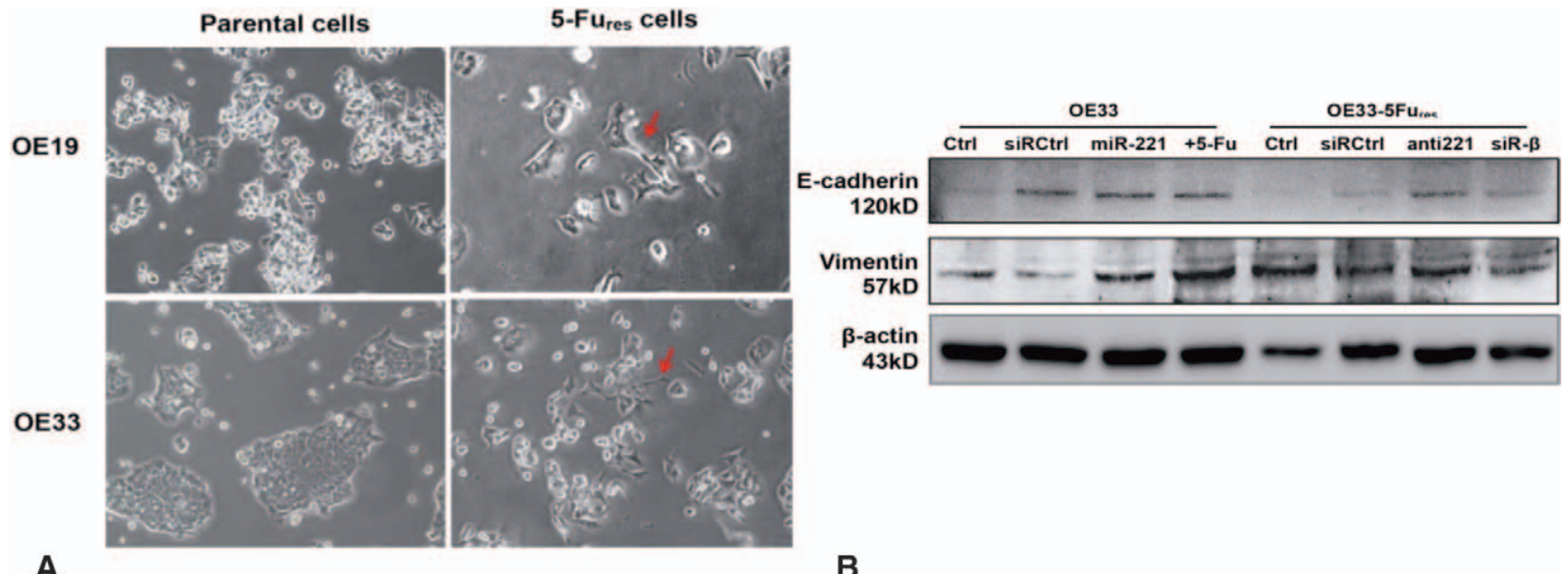

A

B
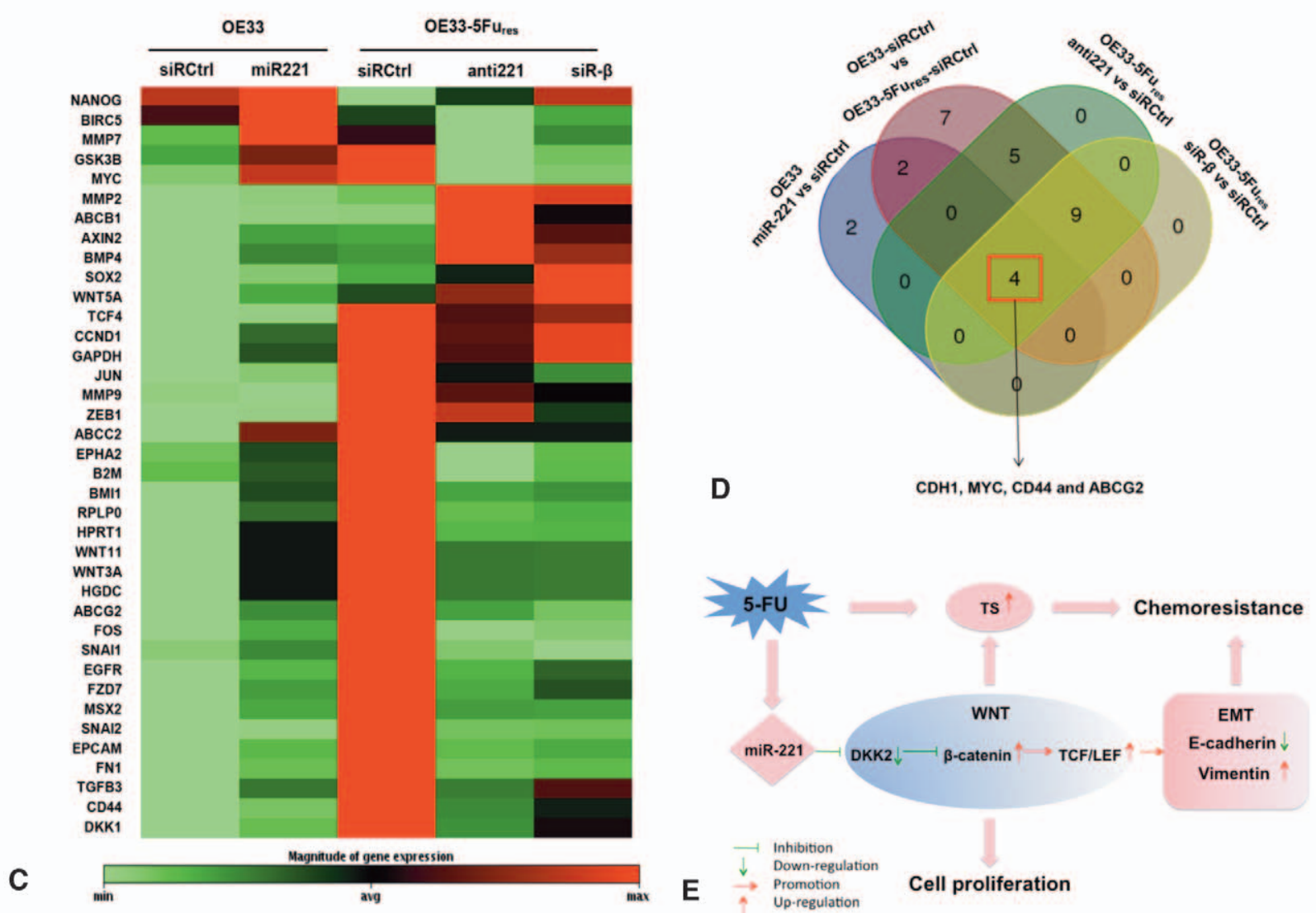

FIGURE 5. Effects of miR-221 on the interaction of WNT/ $\beta$-catenin signaling and EMT in EC cells. A, Morphological comparison of 5-FU sensitive and resistant EC cells: spindle-like phenotype in 5-FU resistant OE19 and OE33 cells. B, EMT-related markers E-cadherin and Vimentin are regulated by miR-221 in OE33 and OE33-5Fu res cells. C, D, EMT-related Wnt/ $\beta$-catenin target genes are expressed differently in OE33 and OE33-5Fu res cells following transfection with miR-221 mimics, inhibitor or siR-CTNNB1. Four genes $C D H 1, M Y C, C D 44$, and $A B C G 2$ were regulated by both miR-221 and $\beta$-catenin. $E$, The above results suggest that miR221 acts as a driver of 5-FU resistance in EC. A potential signaling axis between miR-221-DKK $2-W N T / \beta$-catenin-EMT was identified in EC. MiR-221 activates the WNT/ $\beta$-catenin pathway by direct targeting of $\mathrm{DKK}_{2}$ (an antagonist of the WNT/ $\beta$-catenin pathway), leading to enhanced cell proliferation. Activation of the WNT/ $\beta$-catenin pathway leads to accumulation of TS with increase of chemoresistance and epithelial-mesenchymal transition by regulating its downstream targets (c-myc, E-cadherin, CD44, and $A B C G 2)$. 
cancer resistance to fulvestrant by acting through multiple signaling pathways. ${ }^{34}$ In a previous study, we showed that combined suppression of miR-21 and miR-221 sensitized pancreatic cancer cells to treatment with either gemcitabine or 5-FU. Accordingly, our finding that miR-221 is overexpressed in EC cells prompted us to speculate an association between miR-221 and chemotherapy resistance in EC. ${ }^{20}$ In general, chemotherapy regimens for EC are 5-FU based. Hummel et $\mathrm{al}^{37}$ used an in vitro model of acquired chemotherapy resistance in esophageal adeno- and squamous cell carcinoma cells showing miRNA expression profiles for cisplatin or 5-FU resistant variants versus chemotherapy-sensitive controls and found miR-27b3p, miR-193b-3p, miR-192-5p, miR-378 a-3p, miR-125a-5p, and miR-18a-3p dysregulated.

In this study, we show that expression of miR-221 is associated with 5-FU resistance and EC tumor growth. Furthermore, we demonstrate that increased expression of miR-221 correlates with activation of $\mathrm{Wnt} / \beta$-catenin signaling and EMT in EC cells. The Wnt $/ \beta$ catenin signaling pathway is indeed linked to cancer progression and chemoresistance in various tumors via EMT. ${ }^{38-40}$ An antagonist of the Wnt/ $\beta$-catenin pathway is $\mathrm{DKK}_{2},{ }^{41}$ a member of the Dickkopf family, which is activated by treatment with $5-\mathrm{FU} \cdot{ }^{42,43}$ We thus speculated that miR-221 might account for 5-FU resistance in EC by modulation of $\mathrm{DKK}_{2}$ expression. To address this, we examined the expression of $\mathrm{DKK}_{2}$ together with the distribution of $\beta$-catenin in EAC tumor specimens. Our immunohistochemistry analysis demonstrated no significant difference in $\mathrm{DKK}_{2}$ expression in Barrett's mucosa or EC tumor tissue before chemotherapy. We speculate that the missing statistical significance of the correlation analysis between $\mathrm{DKK}_{2}, \beta$ catenin and chemotherapy might be a consequence of limited sample size. Interestingly, esophageal tumor specimens following prolonged exposure to chemotherapy showed a substantial decrease in $\mathrm{DKK}_{2}$ expression together with an accumulation of nuclear $\beta$-catenin (Fig. 4E), while inactivated $\beta$-catenin was mainly localized to the cell membrane and cytoplasm in Barrett's mucosa and EAC specimens without prior chemotherapy treatment. These results suggest that miR221 influence chemoresistance at least in part through activation of the $\mathrm{Wnt} / \beta$-catenin pathway via regulation of $\mathrm{DKK}_{2}$ expression.

In correlation to changes of miR-221 expression, we further identified significant alterations in expression levels of $\beta$-catenin/ Wnt- and EMT-associated genes such as $M Y C, C D 44, C D H 1$, and $A B C G 2$. We therefore propose that increased expression of miR-221 leads to reduced expression of $\mathrm{DKK}_{2}$, further releasing an activation of the $\mathrm{Wnt} / \beta$-catenin pathway resulting in nuclear translocation of $\beta$ catenin with corresponding expression of Wnt target genes.

Accordingly, our results suggest that miR-221 functions as an oncomiR in EAC. Moreover, the present study is the first to connect $\mathrm{Wnt} / \beta$-catenin signaling and EMT-related gene expression in association to 5-FU resistance of EC. The potential mechanism for this general phenomenon is summarized in Fig. 5E.

Chemoresistance is a major issue for effective treatment of EC. On the basis of our results, increased miR-221 could either act as a surrogate marker to predict chemotherapy resistance or as a potential therapeutic target against chemotherapy resistance in EAC.

The results show a decrease in the expression of $\mathrm{DKK}_{2}$ in esophageal tumor tissues, and nuclear accumulation of $\beta$-catenin in 5-FU resistant EC cells, and in tumor samples linked to a poor response to 5-FU therapy. The data suggest a tendency toward activation of the Wnt/ $\beta$-catenin pathway in EAC that is associated with long time exposure to anticancer drugs especially 5-FU. However, due to the limitation of acquiring more EAC tissues from either local or independent clinical centers, we expected this finding could be further confirmed in other studies.

We further validated significant changes in the expression levels of $\beta$-catenin/Wnt- and chemotherapy-associated genes $M Y C$,
$C D 44, C D H 1$, and $A B C G 2$ in response to changes in miR-221 expression. On the basis of our results, we propose that increased expression of miR-221 leads to reduced expression of $\mathrm{DKK}_{2}$, further releasing a DKK2-mediated blockade of the Wnt/ $\beta$-catenin signaling pathway. Increased activation of this pathway results in disruption of the Axin/APC/GSK3 degradation complex leading to stabilization of $\beta$-catenin. $\beta$-catenin then translocates to the nucleus wherein it helps control expression of Wnt targets.

Our results suggest that miR-221 functions as an oncomiR in EAC by direct targeting of $\mathrm{DKK}_{2}$, as evidenced by the associated effects observed on the $\mathrm{Wnt} / \beta$-catenin signaling pathway. Processes linked to EMT resulted from the continuous 5-FU treatment of EAC cell lines. EMT has been previously associated with 5-FU resistance $^{44-46}$ and miR-221 in pancreatic cancer cells. ${ }^{47}$

The present study is the first to connect $\mathrm{Wnt} / \beta$-catenin pathway and the process of EMT in 5-FU resistance of EAC. The potential mechanism for this general phenomenon is summarized in Fig. 5E.

Chemoresistance is a major issue in the effective treatment of EAC. Thus, detecting rational biomarkers to predict chemotherapy sensitivity and screening for targets to overcome resistance are significant for cancer therapy. On the basis of our results, increased miR-221 expression is associated with chemotherapy resistance and poor prognosis. MiR-221 may thus act as a surrogate marker to predict chemoresistance in EAC.

\section{ACKNOWLEDGMENTS}

We thank Prof. Christian Mawrin, Department of Neuropathology, Otto-von-Guericke University, Magdeburg, Germany for support and assistance. We also thank Anneli Tischmacher for excellent technology support.

\section{REFERENCES}

1. Parkin DM, Bray F, Ferlay J, et al. Global cancer statistics, 2002. CA Cancer J Clin. 2005;55:74-108.

2. Gebski V, Burmeister B, Smithers BM, et al. Survival benefits from neoadjuvant chemoradiotherapy or chemotherapy in oesophageal carcinoma: a meta-analysis. Lancet Oncol. 2007;8:226-234.

3. Straatman J, Joosten PJ, Terwee CB, et al. Systematic review of patientreported outcome measures in the surgical treatment of patients with esophageal cancer. Dis Esophagus. 2015 [Epub ahead of print].

4. Kelsen DP, Ginsberg R, Pajak TF, et al. Chemotherapy followed by surgery compared with surgery alone for localized esophageal cancer. $N$ Engl J Med. 1998;339:1979-1984.

5. Montgomery EA, et al. Oesophageal cancer. In: Stewart BW, Wild CP, eds World Cancer Report. 2014:528-543.

6. Zhang HZ, Jin GF, Shen HB. Epidemiologic differences in esophageal cancer between Asian and Western populations. Chin J Cancer. 2012;31:281-286.

7. Bartel DP. MicroRNAs: genomics, biogenesis, mechanism, and function. Cell. 2004;116:281-297.

8. Calin GA, Croce CM. MicroRNA signatures in human cancers. Nat Rev Cancer. 2006;6:857-866.

9. Gottesman MM. Mechanisms of cancer drug resistance. Annu Rev Med. 2002;53:615-627.

10. Gatti L, Zunino F. Overview of tumor cell chemoresistance mechanisms. Methods Mol Med. 2005;111:127-148.

11. Wilson TR, Longley DB, Johnston PG. Chemoresistance in solid tumours. Ann Oncol. 2006;17(Suppl 10):x315-x324.

12. Ullah MF. Cancer multidrug resistance (MDR): a major impediment to effective chemotherapy. Asian Pac J Cancer Prev. 2008;9:1-6.

13. Fletcher JI, Haber M, Henderson MJ, et al. ABC transporters in cancer: more than just drug efflux pumps. Nat Rev Cancer. 2010;10:147-156.

14. Imanaka Y, Tsuchiya S, Sato F, et al. MicroRNA-141 confers resistance to cisplatin-induced apoptosis by targeting YAP1 in human esophageal squamous cell carcinoma. J Hum Genet. 2011;56:270-276.

15. Hamano R, Miyata H, Yamasaki M, et al. Overexpression of miR-200c induces chemoresistance in esophageal cancers mediated through activation of the Akt signaling pathway. Clin Cancer Res. 2011; 17:3029-3038 
16. Hummel R, Watson DI, Smith C, et al. Mir-148a improves response to chemotherapy in sensitive and resistant oesophageal adenocarcinoma and squamous cell carcinoma cells. J Gastrointest Surg. 2011;15:429-438.

17. Hong L, Han Y, Zhang H, et al. The prognostic and chemotherapeutic value of miR-296 in esophageal squamous cell carcinoma. Ann Surg. 2010;251:10561063.

18. Zhang H, Li M, Han Y, et al. Down-regulation of miR-27a might reverse multidrug resistance of esophageal squamous cell carcinoma. Dig Dis Sci. 2010;55:2545-2551

19. Zhao Y, Bao Q, Schwarz B, et al. Stem cell-like side populations in esophageal cancer: a source of chemotherapy resistance and metastases. Stem Cells Dev. 2014;23:180-192.

20. Zhao Y, Zhao L, Ischenko I, et al. Antisense inhibition of microRNA-21 and microRNA-221 in tumor-initiating stem-like cells modulates tumorigenesis, metastasis, and chemotherapy resistance in pancreatic cancer. Target Oncol. 2015; $10: 535-548$

21. Herbst A, Jurinovic V, Krebs S, et al. Comprehensive analysis of beta-catenin target genes in colorectal carcinoma cell lines with deregulated Wnt/betacatenin signaling. BMC Genomics. 2014;15:74.

22. Wang CM, Wang Y, Fan CG, et al. miR-29c targets TNFAIP3, inhibits cell proliferation and induces apoptosis in hepatitis B virus-related hepatocellular carcinoma. Biochem Biophys Res Commun. 2011;411:586-592.

23. Crea F, Danesi R, Farrar WL. Cancer stem cell epigenetics and chemoresistance. Epigenomics. 2009;1:63-79.

24. O'Connor ML, Xiang D, Shigdar S, et al. Cancer stem cells: a contentious hypothesis now moving forward. Cancer Lett. 2014;344:180-187.

25. Reya T, Morrison SJ, Clarke MF, et al. Stem cells, cancer, and cancer stem cells. Nature. 2001;414:105-111.

26. Takahashi RU, Miyazaki H, Ochiya T. The role of microRNAs in the regulation of cancer stem cells. Front Genet. 2014;4:295.

27. Mathieu J, Ruohola-Baker H. Regulation of stem cell populations by microRNAs. Adv Exp Med Biol. 2013;786:329-351.

28. Zhao G, Cai C, Yang T, et al. MicroRNA-221 induces cell survival and cisplatin resistance through PI3K/Akt pathway in human osteosarcoma. PLoS One. 2013;8:e53906.

29. Yuan K, Xie K, Fox J, et al. Decreased levels of miR-224 and the passenger strand of miR-221 increase MBD2, suppressing maspin and promoting colorectal tumor growth and metastasis in mice. Gastroenterology. 2013;145:853-864.

30. Dahiya N, Sherman-Baust CA, Wang TL, et al. MicroRNA expression and identification of putative miRNA targets in ovarian cancer. PLoS One. 2008;3:e2436.

31. Wei Y, Lai X, Yu S, et al. Exosomal miR-221/222 enhances tamoxifen resistance in recipient ER-positive breast cancer cells. Breast Cancer Res Treat. 2014;147:423-431.

32. Stanitz E, Juhasz K, Gombos K, et al. Alteration of miRNA expression correlates with lifestyle, social and environmental determinants in esophageal carcinoma. Anticancer Res. 2015;35:1091-1097.

33. Chun-Zhi Z, Lei H, An-Ling Z, et al. MicroRNA-221 and microRNA-222 regulate gastric carcinoma cell proliferation and radioresistance by targeting PTEN. BMC Cancer. 2010;10:367.

34. Rao X, Di Leva G, Li M, et al. MicroRNA-221/222 confers breast cancer fulvestrant resistance by regulating multiple signaling pathways. Oncogene. 2011;30:1082-1097.

35. Xie Q, Yan Y, Huang Z, et al. MicroRNA-221 targeting PI3-K/Akt signaling axis induces cell proliferation and BCNU resistance in human glioblastoma. Neuropathology. 2014;34:455-464.

36. Zhang C, Kang C, Wang P, et al. MicroRNA-221 and -222 regulate radiation sensitivity by targeting the PTEN pathway. Int J Radiat Oncol Biol Phys. 2011;80:240-248.

37. Hummel R, Sie C, Watson DI, et al. MicroRNA signatures in chemotherapy resistant esophageal cancer cell lines. World J Gastroenterol. 2014;20:1490414912.

38. Hiscox S, Jiang WG, Obermeier K, et al. Tamoxifen resistance in MCF7 cells promotes EMT-like behaviour and involves modulation of beta-catenin phosphorylation. Int J Cancer. 2006;118:290-301.

39. Huynh TT, Rao YK, Lee WH, et al. Destruxin B inhibits hepatocellular carcinoma cell growth through modulation of the Wnt/beta-catenin signaling pathway and epithelial-mesenchymal transition. Toxicol In Vitro. 2014;28:552-561.

40. Gerard B, Tait L, Nangia-Makker P, et al. Rad6B acts downstream of Wnt signaling to stabilize beta-catenin: implications for a novel Wnt/beta-catenin target. J Mol Signal. 2011;6:6.

41. Kawano Y, Kypta R. Secreted antagonists of the Wnt signalling pathway. J Cell Sci. 2003;116(Pt 13):2627-2634.
42. Noda T, Nagano H, Takemasa I, et al. Activation of Wnt/beta-catenin signalling pathway induces chemoresistance to interferon-alpha/5-fluorouracil combination therapy for hepatocellular carcinoma. Br J Cancer. 2009;100:1647-1658.

43. Chikazawa N, Tanaka H, Tasaka T, et al. Inhibition of Wnt signaling pathway decreases chemotherapy-resistant side-population colon cancer cells. Anticancer Res. 2010;30:2041-2048.

44. Arumugam T, Ramachandran V, Fournier KF, et al. Epithelial to mesenchymal transition contributes to drug resistance in pancreatic cancer. Cancer Res. 2009;69:5820-5828.

45. Findlay VJ, Wang C, Nogueira LM, et al. SNAI2 modulates colorectal cancer 5-fluorouracil sensitivity through miR145 repression. Mol Cancer Ther. 2014;13:2713-2726.

46. Toden S, Okugawa Y, Jascur T, et al. Curcumin mediates chemosensitization to 5-fluorouracil through miRNA-induced suppression of epithelial-to-mesenchymal transition in chemoresistant colorectal cancer. Carcinogenesis. 2015;36:355-367

47. Su A, He S, Tian B, et al. MicroRNA-221 mediates the effects of PDGF-BB on migration, proliferation, and the epithelial-mesenchymal transition in pancreatic cancer cells. PLoS One. 2013;8:e71309.

\section{DISCUSSANTS}

\section{Bonavina (Milano, Italy):}

Tumor heterogeneity and chemoresistance remain indeed a main issue for an effective neo-adjuvant therapy in esophageal adenocarcinoma. 5-FU has been a mainstay of treatment for a long time, but most clinical studies have focused on speed of drug delivery (whether bolus or continuous) and on the enzimatic defects associated with 5-FU toxicity rather than on chemoresistance.

You have described a large set of experiments showing that miR-221 positive tumor cells have more malignant potential and are more resistant to 5-FU than naive tumor cells. You have also shown that miR-221 may serve as a prognostic marker and as a therapeutic target for patients with 5-FU resistant esophageal adenocarcinoma.

To be considered an efficient prognostic marker, miR-221 needs further validation in clinical trials. In addition, the possibility to sample blood or other body fluids should be investigated to assess specificity and sensitivity in esophageal adenocarcinoma.

My questions for you are the following: First, is there any possible relationship between speed of 5-FU delivery and induction of chemoresistance? Second, is there any association between the risk of 5-FU toxicity and 5-FU chemoresistance? Third, would you speculate that knockdown of miR-221 to restore chemosensitivity to 5-FU may be the winning strategy in the future or rather that it may be more convenient to focus on the more innovative targeted drugs?

\section{Response From C. Bruns (Cologne, Germany):}

With respect to the possible relationship of the speed of 5-FU delivery or continuous versus bolus 5-FU application, all analyzed specimen derived from patients who received continuous 5-FU injection within their chemotherapy protocol. However, I think that indeed long-term, continuous exposure to 5-FU-here in the experimental setting even with increasing doses-induces resistance mechanisms and select for cell populations that are highly resistant. Transferred to clinical situations, protocols with short-term exposure to chemotherapy should rather be conducted.

With respect to second question, we did not find any association between 5-FU toxicity and 5-FU resistance. 5-FU toxicity is based on a DPD mutation and we have not investigated the cell lines regarding their DPD mutational status.

With respect to the last question, we indeed speculate that possible new techniques to target resistant esophageal 
adenocarcinoma would be specific miRNA aside of being markers for resistance. Another option would be to target downstream molecular markers or pathways associated with specific miR-RNAs. As miR-221 is activating the wnt-pathway, it would be interesting to think of wnt-inhibitors as therapeutic targets of resistant esophageal adenocarcinoma. A well-known wnt-inhibitor is aspirin, which is already been known to interfere in GI malignancies with the adenoma-carcinoma sequence.

\section{J.V. Reynolds (Dublin, Ireland):}

miR-221, in addition to being an oncogene, is also a known tumor-suppressor gene and low expression levels or downregulation are implicated, for example, in the development of oropharyngeal SCCs. It also downregulates the c-kit receptor, so it is implicated in the development of hemopoietic malignancies. Did you notice when you knocked down miR-221 expression that it induced development of SCCs in the oropharynx or did you check in your mice if there were any erthyropoietic maligancies as a consequence of that?
Finally, do you have plans to measure miR-221 in preand post-neoadjuvant chemoradiotherapy esophageal biopsies to see if expression levels change in response to effective treatment?

\section{Response From C. Bruns (Cologne, Germany):}

No, we did not check our animals for development of oropharyngeal SCCs.

However, we analyzed commercially available esophageal SCC cell lines and found the expression of miR-RNA 221 in these esophageal SCC cell lines not that impressive.

With respect to your second question, we have not yet analyzed pre- and post-neoadjuvant chemoradiotherapy esophageal biopsies. We only analyzed full histology samples after esophageal tumor resection. In the future, this would of course be the correct way to identify miR-RNA 221 expression in tumor biopsies to individualize chemotherapy for each patient. 